\title{
Spondylocarpotarsal synostosis
}

INSERM

\section{Source}

INSERM. (1999). Orphanet: an online rare disease and orphan drug data base.

Spondylocarpotarsal synostosis. ORPHA:3275

Spondylocarpotarsal synostosis (SCT) syndrome is a skeletal dysplasia clinically

characterized by postnatal progressive vertebral fusions frequently manifesting as block

vertebrae, contributing to an undersized trunk and a disproportionate short stature,

scoliosis, lordosis, carpal and tarsal synostosis, with club feet and a mild facial

dysmorphism. 